\title{
Dendritic cell factor 1 inhibits proliferation and migration and induces apoptosis of neuroblastoma cells by inhibiting the ERK signaling pathway
}

\author{
GUANGHONG LUO*, RUILI FENG* , YANGYANG SUN, LILI ZHENG, \\ YAJIANG WANG, YANLU CHEN and TIEQIAO WEN
}

Laboratory of Molecular Neural Biology, School of Life Sciences, Shanghai University, Shanghai 200444, P.R. China

Received December 1, 2017; Accepted October 9, 2018

DOI: $10.3892 /$ or.2018.6796

\begin{abstract}
Neuroblastoma (NB) is the most common extracranial solid tumor that affects mainly children and has extremely high mortality and recurrence rates. A previous study revealed that dendritic cell factor 1 (DCF1), also called transmembrane protein 59, could activate apoptosis in glioma cells. In the present study, we applied immunofluorescence, western blot analysis, flow cytometry and cell tumorigenicity to investigate the DCF1 mechanisms involved in NB apoptosis. DCF1 was overexpressed in Neuro-2a and SK-N-SH cells through instantaneous transfection. The data revealed that overexpression of DCF1 could inhibit cell proliferation, migration, invasion and promote cell apoptosis in vitro, and suppress NB growth in vivo. The ERK1/2 signaling pathway, which promotes cell survival, was the target of DCF1 in neuroblastoma cells. All the results indicated that DCF1 could be a potential therapeutic target for the understanding and treatment of NB.
\end{abstract}

\section{Introduction}

Cancer is the leading cause of death among children aged 1-14 years in the United States, and neuroblastoma (NB) accounts for $6 \%$ of those patients (1). NB is a common solid extracranial tumor in children that is diagnosed as a metastatic disease, most often metastasizing to the bone, bone marrow and lymph nodes $(2,3)$ which destroys the body's immune system. Despite the latest methods of chemotherapy and surgery, this type of tumor continues to make the prognosis very dismal for children presenting with advanced stages of the disease. Only 18-30\% of patients with this type of cancer survive (4). Even worse, the

Correspondence to: Professor Tieqiao Wen, Laboratory of Molecular Neural Biology, School of Life Sciences, Shanghai University, 381 Nanchen Road, Shanghai 200444, P.R. China

E-mail:wtq@shu.edu.cn

${ }^{*}$ Contributed equally

Key words: DCF1, ERK1/2 signaling pathway, Neuro-2a, neuroblastoma, apoptosis mortality and recurrence rates of patients with high-risk (HR) $\mathrm{NB}$ is $>60 \%$ (5), which is a heavy burden on both the patients and their families. An NB cell is a poorly differentiated type of cancer cell; therefore, it contains the functional and molecular characteristics of normal stem cells, which enables it to evade growth suppressors (6) that would increase patient survival. These characteristics provide NB with the ability to resist chemo- and radiotherapy treatments (7). In general, the overexpression of special cell-survival pathways and a deficiency in normal cell senescence or apoptosis control contribute to NB tumorigenesis and malignant transformation; therefore, the breakdown or hindrance in the connection to cell-survival pathways may decrease the potential for NB malignancy and provide several avenues to explore new NB therapies.

The Ras/Raf/MEK/ERK cascade pathway has been reported to play important roles in cancer cell growth and NB survival (8-10). Active mutations and/or overexpression of various components within this pathway (EGFR, Ras and Raf) are frequently observed in NB $(11,12)$, especially in relapsing NB $(13,14)$. As such, researchers have focused on the Raf/MEK/ERK pathway in intense studies to identify new target-based approaches for cancer treatment to obtain more favorable results (15-17). Unfortunately, the disease in a majority of NB patients who receive treatment with Ras, Raf and MEK inhibitors becomes resistant after $\sim 6-8$ months and the drugs exhibit significantly reduced clinical efficacy. In view of this, the ERK1/2 pathway may be one of the best targets for NB treatment (17).

Previous studies have revealed that DCF1 plays a vital role in various cell processes, including regulation of neural stem-cell proliferation and differentiation $(18,19)$, glioma cell apoptosis (20) and dendritic spine formation (21) and maintaining energy balance (22). Xie et al (23) demonstrated that DCF1 was significantly alerted among different grades of tumors and that DCF1 overexpression led to apoptosis by causing the cell mitochondria to dysfunction.

In the present study, we confirmed that the DCF1 inhibited cell viability and motility, promoted the apoptosis of Neuro-2a (N2a) and SK-N-SH cells, and identified the molecular mechanism by which DCF1 exerts tumor-suppressive effects on NB cells by inhibiting the ERK1/2 signaling pathway to promote apoptosis. 


\section{Materials and methods}

All experiments were approved and performed in accordance with the guidelines and regulations of the Shanghai University Ethics Committee (Shanghai, China).

Plasmid construction. Human $d c f 1$ was amplified from the 293T cellular cDNA library using polymerase chain reaction (PCR) with KOD-Plus-Neo DNA polymerase (cat. no. KOD-401; Toyobo Shanghai Biotech Co., Ltd., Shanghai, China) and cloned into the $\mathrm{XhoI}$ and $\mathrm{SmaI}$ restriction sites of the pCAGGSEGFP plasmid and into the HindIII and XhoI restriction sites of the pcDNA3.1-myc-his plasmid using following primers: sense, 5'-CGGAATTCATGGCGGCGCCGAAGGGGAG-3' andantisense, 5'-CGGGATCCGTAAAATTTCAGAATGAGCA-3'. The small interfering RNA plasmid of DCF1 (psi-DCF1) was preserved in our laboratory.

Cell culture. The N2a mouse brain neuroma cells were purchased from the Cell Bank of the Type Culture Collection of the Chinese Academy of Sciences (Shanghai, China). $\mathrm{N} 2 \mathrm{a}$ cells were cultured according to the methods used in a previous study (24). In brief, N2a cells were grown in an incubator containing Minimum Essential Medium (MEM) (cat. no. 32561094; Thermo Fisher Scientific, Shanghai, China) supplemented with GlutaMAX, $10 \%$ fetal bovine serum (FBS) (cat. no. 10100147; Thermo Fisher Scientific) and $100 \mathrm{U} / \mathrm{ml}$ penicillin/streptomycin (cat. no. 10378016; Thermo Fisher Scientific) in $5 \% \mathrm{CO}_{2}$ at $37^{\circ} \mathrm{C}$. SK-N-SH cells were purchased from FU Heng Biology (cat. no. FH0164; FuHeng Cell Center, Shanghai, China). SK-N-SH cells were grown in an incubator containing Dulbecco's modified Eagle's medium (DMEM) supplemented with $10 \% \mathrm{FBS}$ and $100 \mathrm{U} / \mathrm{ml}$ penicillin/streptomycin in $5 \% \mathrm{CO}_{2}$ at $37^{\circ} \mathrm{C}$. The culture medium was changed every 1-2 days. For all experiments, the cells were grown to $\sim 90 \%$ confluence and subjected to no more than 20 cell passages. The cells were harvested and seeded at a density of $2 \times 10^{5}$ cells/well in 12 -well culture plates and used for fluorescence correlation spectroscopy (FCS), western blotting, cell migration assay, Matrigel invasion assay and immunofluorescence after being transfected with plasmid pCAGGS-DCF1-EGFP and pCAGGS-EGFP or pcDNA3.1 and pcDNA3.1-DCF1.

Cell transfection. Cells were plated on 12- or 24-well plates at a density of $2 \times 10^{5}$ cells/well or $1 \times 10^{5}$ cells/well and cultured overnight at $37^{\circ} \mathrm{C}$ in an atmosphere of $5 \% \mathrm{CO}_{2}$ for DNA transfection of up to $70 \%$ confluence. Lipofectamine 2000 (cat. no. 11668019; Thermo Fisher Scientific) or calcium phosphate (cat. no. K278001; Thermo Fisher Scientific) was used according to the manufacturer's protocols to transfect $1 \mu \mathrm{g} / \mathrm{well}$ plasmid into the cells.

Cell proliferation. Cell proliferation after DCF1 transfection was assessed using the Cell Counting Kit-8 (CCK-8; product no. C0038; Beyotime Institute of Biotechnology, Shanghai, China). Briefly, the cells were seeded on 96-well plates for 18-20 $\mathrm{h}$ and transfected with plasmid pcDNA3.1-myc-his or pcDNA3.1-DCF1-myc-his for the appropriate time needed for proliferation. A mixture of $10 \mu \mathrm{l}$ of CCK- 8 and $90 \mu \mathrm{l}$ of serum-free DMEM culture medium solution was added to each well at 12-h intervals. After being incubated with CCK-8 solution for $1 \mathrm{~h}$, the absorbance at $450 \mathrm{~nm}$ was measured using a microplate reader (Bio-Rad Laboratories, Hercules, CA, USA). The ability for the cells to proliferate was characterized by optical absorption, and each experiment was conducted five times.

Wound healing assay. Cell wound healing assays were performed in 6-well tissue culture plates. Cells at 30-40\% confluence were transfected with pcDNA3.1-myc-his or pcDNA3.1-DCF1-myc-his. After $24 \mathrm{~h}$, cell confluence reached $\sim 75 \%$ and scratches in the wells were made using 1-ml pipette tips. The wells were then washed twice with full medium to remove the suspended cells and allowed to grow for an additional $48 \mathrm{~h}$. Images were captured using a Nikon TI-S inverted microscope (Nikon Corp., Tokyo, Japan) at 12-h intervals. The migration gap distance was measured using Image-Pro Plus software (Media Cybernetics, Rockville, MD, USA) and the $\%$ of wound closure was calculated as follows: $\left[\left(\mathrm{A}_{\mathrm{t}=0} \mathrm{~h}-\right.\right.$ $\left.\left.\mathrm{A}_{\mathrm{t}=\Delta \mathrm{h}}\right) / \mathrm{A}_{\mathrm{t}=0 \mathrm{~h}}\right] \times 100 \%$, where, $\mathrm{A}_{\mathrm{t}=0 \mathrm{~h}}$ is the area of the wound measured immediately after scratching, and $\mathrm{A}_{\mathrm{t}=\Delta \mathrm{h}}$ is the area of the wound measured at 12,24, 36 and $48 \mathrm{~h}$ after scratching. When the samples were compared with the control cells, the differences were considered significant if $\mathrm{P}<0.05, \mathrm{P}<0.01$ and $\mathrm{P}<0.001$.

Cell invasion. The effect of DCF1 overexpression on the cells was assessed using the BioCoat ${ }^{\mathrm{TM}}$ Matrigel $^{\mathrm{TM}}$ Invasion Chamber (cat. no. 354480; BD Biosciences, San Jose, CA, USA). N2a and SK-N-SH cells were transfected with pCAGGSEGFP and pCAGGS-DCF1-EGFP for $48 \mathrm{~h}$, after which the cells were harvested and adjusted to a density of $5 \times 10^{4}$ cells $/ \mathrm{ml}$. After incubation in the invasion chamber for $22 \mathrm{~h}$ in a humidified atmosphere of $5 \% \mathrm{CO}_{2}$, the non-invaded cells were scrubbed with a cotton swab, and the remaining transfected cells were stained with $1 \%$ crystal violet and observed under a Nikon Ti-S fluorescence microscope (Nikon Corp.) at an x100 magnification. Each experiment was performed in triplicate.

Cell cycle detection using flow cytometry. After transfection with pcDNA3.1-myc-his or pcDNA3.1-DCF1-myc-his for $48 \mathrm{~h}$, the cells were harvested using trypsin $[0.25 \%$ in phosphate-buffered solution (PBS) solution without ethylenediaminetetraacetic acid (EDTA)]. Next, the cells were washed twice with $2 \%$ bovine serum albumin (BSA)-PBS and resuspended in $200 \mu \mathrm{l}$ cold PBS, to which precooled $100 \%$ ethanol was slowly added dropwise, and the solution was gently mixed to reach $70 \%$ ethanol. The mixture was fixed overnight at $-20^{\circ} \mathrm{C}$, after which $5 \mu \mathrm{l}$ RNase $(10 \mathrm{mg} / \mathrm{ml})$ was added for $30 \mathrm{~min}$ at $37^{\circ} \mathrm{C}$ to digest the RNA. To prepare for flow cytometry, the nuclei were stained in the dark with $100 \mu \mathrm{g} / \mathrm{ml}$ propidium iodide (PI) for $30 \mathrm{~min}$ (Beckman Coulter, Inc., Brea, CA, USA).

Cell apoptosis detection by flow cytometry. After transfection with pcDNA3.1-myc-his or pcDNA3.1-DCF1-myc-his for $48 \mathrm{~h}$, the cells were harvested using trypsin $(0.25 \%$ in PBS solution without EDTA), washed twice with $2 \%$-BSA-PBS, and resuspended in $100 \mu \mathrm{l}$ binding buffer combined with 
$5 \mu \mathrm{l}$ Annexin V-FITC and gently mixed. Next, $10 \mu 1$ 7-AAD was added to stain the cells according to the manufacturer's protocol for the Annexin V Apoptosis Detection kit with 7-AAD (BioLegend, San Diego, CA, USA). Samples were stained for $15 \mathrm{~min}$ at room temperature (RT), after which $400 \mu \mathrm{l}$ binding buffer was added to prepare the samples for flow cytometry detection (Beckman Coulter, Inc.).

Cell tumorigenicity in nude mice. BALB/c athymic nude mice (Shanghai SLAC Laboratory Animal Co., Ltd., Shanghai, China; 6-8 weeks of age, male) were used, and each experimental group consisted of 4 mice (the control group mice were infected subcutaneously with cells that were transfected with pCAGGS-EGFP, and the experimental group mice were infected subcutaneously with cells that were transfected with pCAGGS-DCF1-EGFP). All animal procedures were carried out in accordance with the guidelines and regulations of the Shanghai University Ethics Committee. Briefly, N2A cells were transfected with pCAGGS-EGFP (control) or pCAGGSDCF1-EGFP for $48 \mathrm{~h}$ and harvested using trypsin digestion. Single cell suspensions were prepared, after which $2 \times 10^{6}$ cells were injected subcutaneously into the right flanks of mice. The mice were raised in the same environment with a controlled temperature $\left(23 \pm 1^{\circ} \mathrm{C}\right)$ and humidity $(40-45 \%)$ on a 12 -h light/dark cycle (08:00-20:00) with enough food and water provided and were observed every week. The tumor volume was calculated as the length $\mathrm{x}$ width $^{2} \mathrm{x} 0.52(25)$.

Immunofluorescence. Graft tumors removed from the nude mice were fixed in precooled $4 \%$ paraformaldehyde in $0.1 \mathrm{M}$ PBS (pH 7.4). After fixing, the tumors were washed with PBS, dehydrated with 20 and $30 \%$ sucrose, frozen in optimal cutting temperature (O.C.T.) compound (Sakura Finetek US Inc., Torrance, CA, USA) and sliced into $20-\mu \mathrm{m}$ sections using a cryostat (Thermo Fisher Scientific, Inc., Waltham, MA, USA). Sections were permeabilized with $0.1 \%$ Triton X-100 for $10 \mathrm{~min}$ and then washed three times with PBS. The slices were blocked with 5\% BSA in PBS for $60 \mathrm{~min}$ and incubated with primary antibodies Tuj1 (dilution 1:500; cat. no. ab18207; Abcam Biotechnology, Cambridge, UK) and Nestin (dilution 1:100; cat. no. sc-23927; Santa Cruz Biotechnology, Inc., Santa Cruz, CA, USA) overnight at $4^{\circ} \mathrm{C}$. After washing three times with PBS, the slices were incubated with secondary antibodies [cat. no. ZF-0513; Alexa Fluor ${ }^{\circledR}$ 594-conjugated goat anti-mouse IgG (H+L); cat. no. ZF-0511; Alexa Fluor ${ }^{\circledR}$ 488-conjugated goat anti-mouse $\operatorname{IgG}(\mathrm{H}+\mathrm{L})$; Beijing Zhongshan Golden Bridge Biotechnology, Beijing, China] for $60 \mathrm{~min}$ at RT. Finally, the nucleus was stained with 4',6-diamidino-2-phenylindole (DAPI) for $10 \mathrm{~min}$, and fluorescence was detected using the Zeiss LSM 710 confocal microscope (Carl Zeiss, Oberkochen, Germany).

Western blotting. Protein lysates were boiled and subjected to different densities (4-15\%) of sodium dodecyl sulfate (SDS) then transferred onto a nitrocellulose membrane. The membranes were blocked with 5\% BSA or non-fat milk for $1 \mathrm{~h}$ and incubated with the following primary antibodies overnight at $4^{\circ} \mathrm{C}$ : Raf1 (dilution 1:1,000; cat. no. A0223; ABclonal, Wuhan, China), Ras (dilution 1:1,000; cat. no. A12212; ABclonal), Mek1/2 (dilution 1:1,000; cat. no. A11122; ABclonal), p-Erk1/2 (dilution 1:1,000; cat. no. 9101; Cell
Signaling Technology, Danvers, MA, USA), Erk1/2 (dilution 1:1,000; cat. no. 137F5; Cell Signaling Technology), cell cyclin A2 (CCNA2; dilution 1:1,500; cat. no. A7632; ABclonal), cell cyclin kinase 2 (CDK2; dilution 1:1,000; cat. no. A10810; ABclonal); Bax (dilution 1:1,000; cat. no. sc-20067; Santa Cruz Biotechnology, Inc.), BID (dilution 1:1,000; cat. no. sc-373939; Santa Cruz Biotechnology, Inc.), Mcl-1 (dilution 1:1,000; cat. no. sc-53951; Santa Cruz Biotechnology, Inc.), caspase-3 (dilution 1:1,000; cat. no. A2156; ABclonal), survivin (dilution 1:800; cat. no. D221289; Sangon Biotech Co., Ltd., Shanghai, China), Bcl-2 (dilution 1:1,000; cat. no. sc-56015; Santa Cruz Biotechnology, Inc.), glyceraldehyde 3-phosphate dehydrogenase (GAPDH; dilution 1:1,000; cat. no. sc-32233; Santa Cruz Biotechnology, Inc.) and $\alpha$-tubulin (dilution 1:5,000; cat. no. 2144; Cell Signaling Technology, Inc.). The membranes were washed thrice for $5 \mathrm{~min}$ in a mixture of Tris-buffered saline and Polysorbate 20 (TBST), incubated with KPL Dylight 800 goat anti-rabbit immunoglobulin (Ig)G (dilution 1:1,000; cat. no. 5230-0412; KPL, Gaithersburg, MD, USA) or DyLight 700 goat anti-mouse immunoglobulin (Ig)G secondary antibodies (dilution 1:1,000; cat. no. 072-06-18-06; $\mathrm{KPL}$ ) for $1 \mathrm{~h}$, and washed again thrice for $5 \mathrm{~min}$ in TBST. Immunoblotting bands were observed and quantified using the LI-COR Odyssey infrared imaging system (simultaneous twocolor targeted analysis) and software (LI-COR Biosciences, Lincoln, NE, USA).

Statistical analyses. All data were expressed as the mean \pm SEM. Statistical analysis was performed with Independent Student's t-test or one-way ANOVA was used to evaluate the statistical comparison of two and multiple groups, respectively. Tukey's post hoc test was used after the one-way ANOVA. Statistical significance was considered for $\mathrm{P}<0.05, \mathrm{P}<0.01$, $\mathrm{P}<0.001, \mathrm{P}<0.0001$.

\section{Results}

Overexpression of DCF1 inhibits the viability and motility of $N 2 a$ and $S K-N-S H$ cells. To investigate the effect of DCF1 on NB cell growth, N2a cells and SK-N-SH cells were transfected with pcDNA3.1-myc-his or pcDNA3.1-DCF1-myc-his. The result of the CCK-8 assay revealed that DCF1 significantly decreased the cell proliferation rate of N2a (Fig. 1A) and SK-N-SH cells (Fig. 1B). Our previous study indicated that DCF1 inhibited the motility of a glioma cell line (23). In the present study, we conducted invasion and migration assays to detect whether DCF1 inhibited the motility of NB in vitro. We observed that the overexpression of DCF1 could attenuate the invasion (Fig. 1C and D) and migration (Fig. 1E and F) abilities of N2a and SK-N-SH cells compared with cells transfected with pcDNA3.1-myc-his. Therefore, we concluded that DCF1 inhibited the viability and motility of NB cells.

DCF1 has no effect on the cell cycle and cell differentiation of N2a cells in vitro. Since NBs and their derived cell lines maintain the potential to terminally differentiate, and since cell-cycle arrest is tightly coupled to cell differentiation and affects the viability of NB cells, we examined whether DCF1 induced antiproliferative effects through cell-cycle arrest and cell differentiation. To assess this hypothesis, N2a cells were 
A

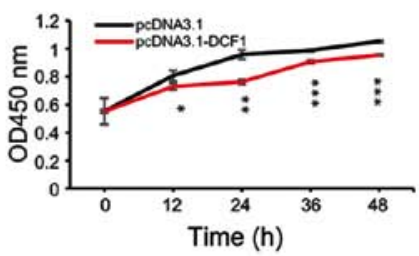

B

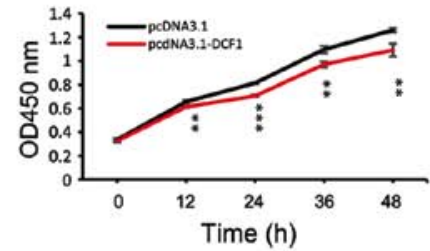

C
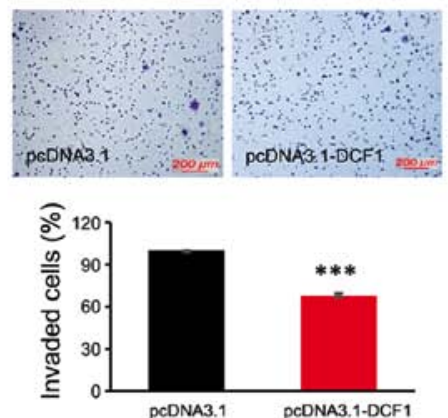

$\mathrm{D}$
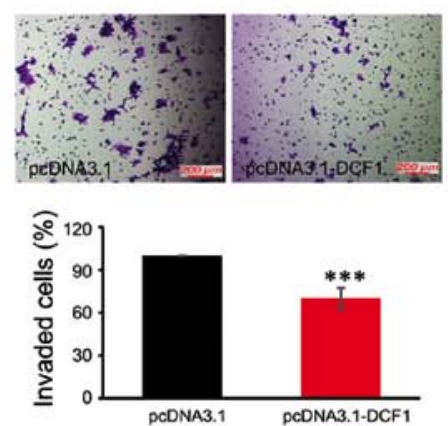

$E$
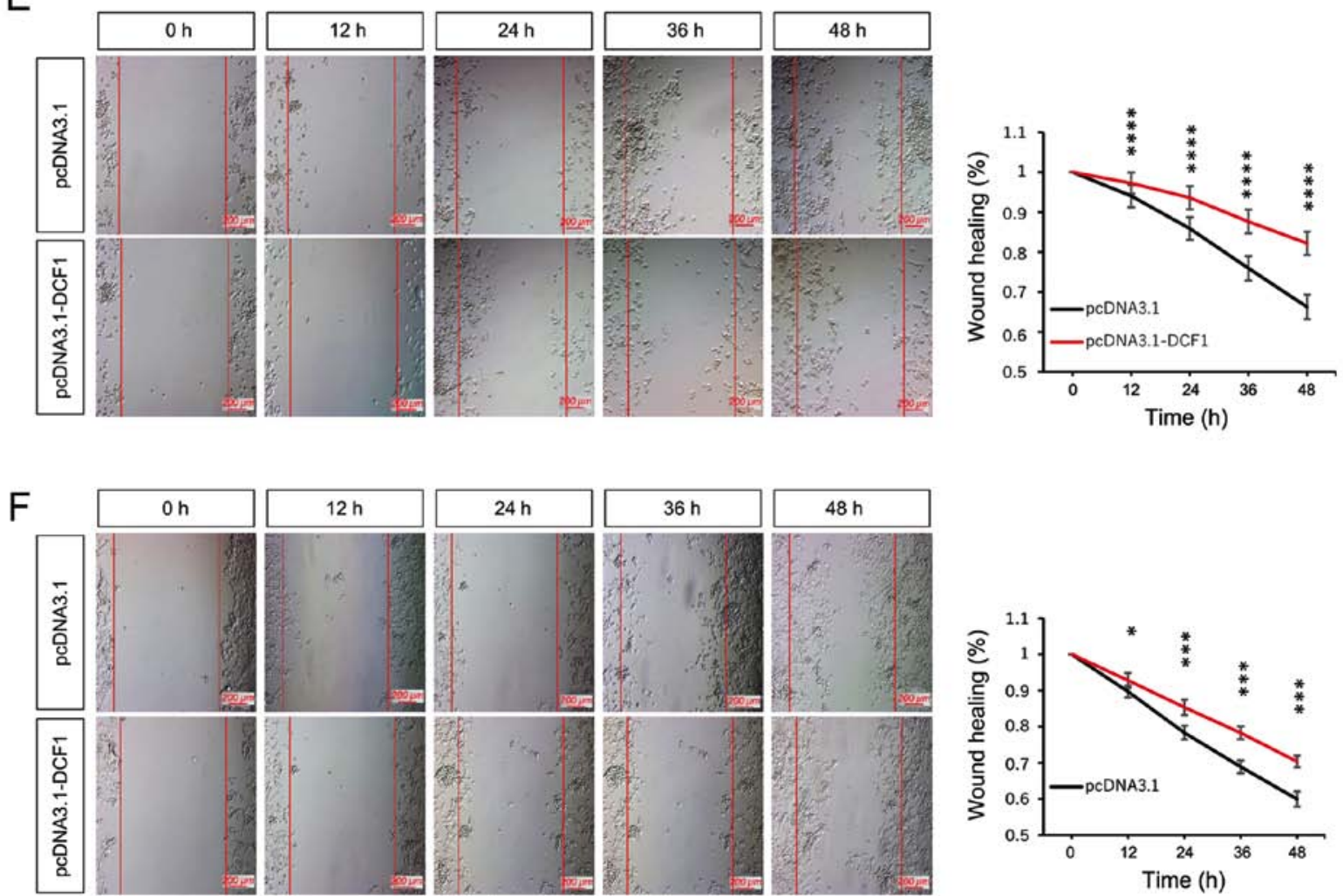

Figure 1. Overexpression of DCF1 inhibits proliferation and motility of NB cells in vitro. N2a and SK-N-SH cells were transfected with pcDNA3.1 and pcDNA3.1-DCF1, respectively. (A) A CCK-8 assay was performed to investigate the effect of DCF1 on the proliferation of N2a cells at different time-points, $\mathrm{n}=5$. (B) A CCK-8 assay was performed to investigate the effect of DCF1 on the proliferation of SK-N-SH cells at different time-points, $n=5$. DCF1 inhibited the proliferation rate. (C) A Transwell assay was used to evaluate the invasion of N2a cells, $n=4$. (D) A Transwell assay was used to evaluate the invasion of SK-N-SH cells, $n=4$. DCF1 decreased the percentage of invasive cells. (E) The migration of N2a cells was evaluated by wound healing assay, $n=3$, quadruplicate. (F) The migration of N2a cells was evaluated by wound healing assay, $n=3$, quadruplicate. DCF1 significantly inhibited the migration rate of NB cells in vitro. ${ }^{*} \mathrm{P}<0.05,{ }^{* * *} \mathrm{P}<0.01,{ }^{* * *} \mathrm{P}<0.001,{ }^{* * * * *} \mathrm{P}<0.0001$. NB, neuroblastoma; DCF1, dendritic cell factor 1; N2a, Neuro-2a; CCK-8, Cell Counting Kit-8.

transfected with either pCAGGS-EGFP or pCAGGS-DCF1EGFP and subsequently processed for fluorescence-activated cell sorting and immunoblotting analysis. The results of cytofluorimetric analysis revealed that the cell cycle was not arrested with DCF1 (Fig. 2A). CCNA2, a cell-cycle protein, controls both the G1/S and G2/M transition phases of the cell cycle and functions by forming specific serine-threonine protein kinase holoenzyme complexes with cyclin-dependent protein kinases, CDK1 or CDK2 (26). CCNA2 was also investigated using immunoblotting. No significant differences were observed (Fig. 2B), which was consistent with the results of the flow cytometric analysis. Next, to examine whether DCF1 induced NB cell differentiation, we monitored the levels of neuronal lineage markers SRY-box 2 (Sox2), Nestin, glial fibrillary acidic protein (GFAP) and neuron-specific Class III $\beta$-tubulin (Tuj1). The expression levels of these markers were not altered after the cells were transfected with DCF1 (Fig. 2C). These results indicated that DCF1 had no effect on the cell cycle or the differentiation of N2a cells.

DCF1 promotes apoptosis of neuroblastoma cells. In view of the fact that DCF1 had no effect on the cell cycle or cell 
A
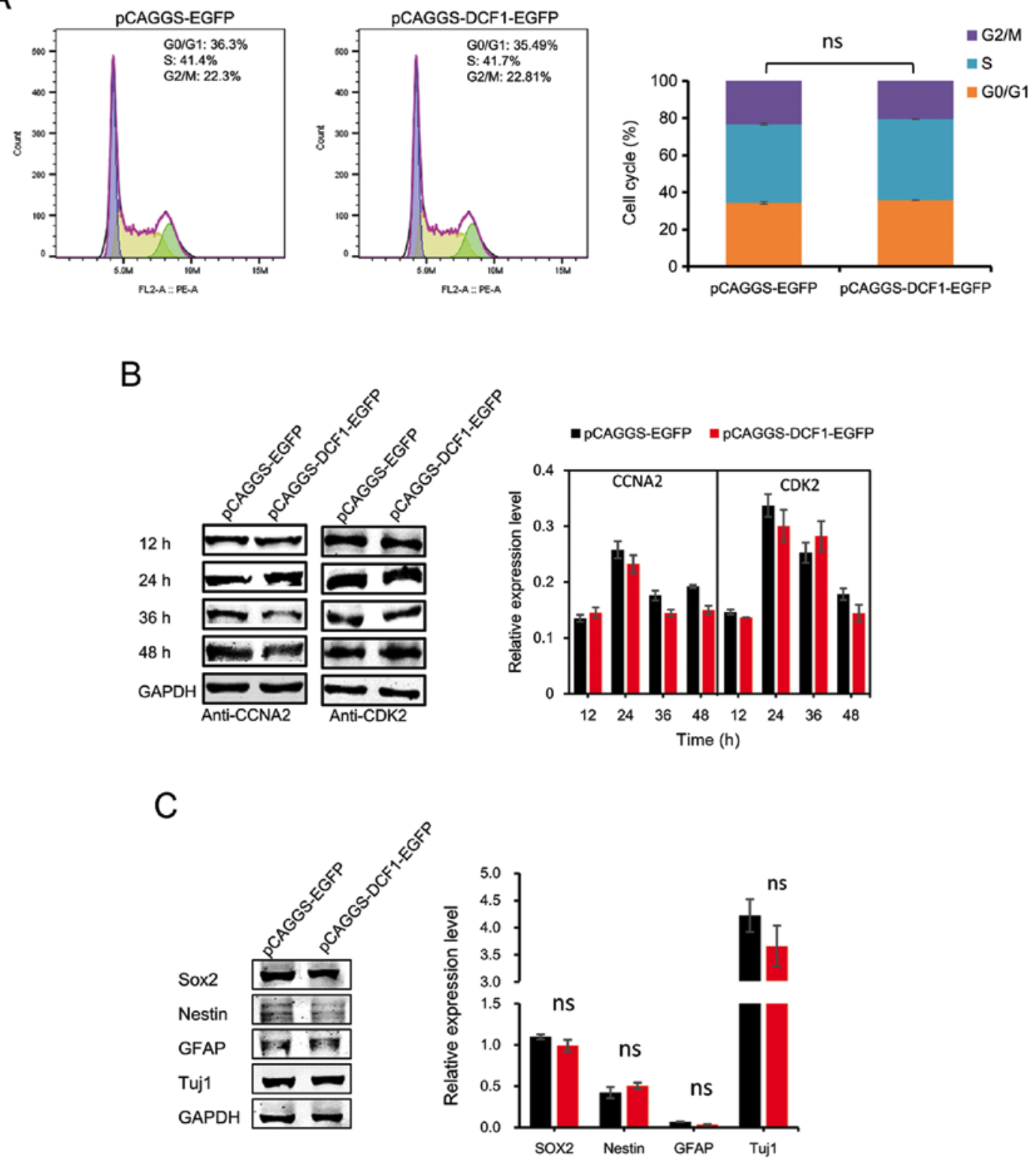

Figure 2. Overexpression of DCF1 has no effect on the cell cycle and cell differentiation of N2a cells in vitro. N2a cells were transfected with pCAGGS-EGFP or pCAGGS-DCF1-EGFP, respectively. (A) N2a cells were analyzed by flow cytometry and quantified DNA distributions by ModFit program, n=6. (B) Cell cycle regulators CCNA2 and CDK2 were examined by western blotting and normalized to GAPDH, $n=4$. (C) Forty-eight hours after transfection, western blot analysis of neuronal differentiation markers, Sox2, Nestin, GFAP and Tuj1 in N2a cells was performed, n=4. DCF1, dendritic cell factor 1; N2a, Neuro-2a; CCNA2, cell cyclin A2; CDK2, cell cyclin kinase 2; Sox2, SRY-box 2; GFAP, glial fibrillary acidic protein; Tuj1, neuron-specific Class III $\beta$-tubulin.

differentiation, we then examined apoptosis, another factor that affects cell viability. After the cells were transfected with pcDNA3.1-myc-his and pcDNA3.1-DCF1-myc-his, respectively, for $48 \mathrm{~h}$, apoptosis in neuroblastoma cells was investigated and analyzed using fluorescence-activated cell detection according to the manufacturer's manual. The results of flow cytometric analysis revealed that the average proportion of apoptotic N2a cells containing pcDNA3.1-DCF1-myc-his (66.67 $\pm 0.58 \%$ ) was significantly higher than those containing pcDNA3.1-myc-his $(24.79 \pm 1.90 \%)$ (Fig. 3A), and that the average proportion of apoptotic SK-N-SH cells transfected with pcDNA3.1-DCF1-myc-his $(59.54 \pm 6.6 \%)$ was higher than those transfected with pcDNA3.1-myc-his $(30.09 \pm 3.6 \%)$ (Fig. 3B). These results indicated that DCF1 had a huge impact on the process of apoptosis in both $\mathrm{N} 2 \mathrm{a}$ and SK-N-SH cells. DAPI staining also revealed an increasing number of $\mathrm{N} 2 \mathrm{a}$ cells that experienced programmed cell death after being transfected with DCF1. The cells with pCAGGS-DCF1-EGFP exhibited more shrinkage in the nucleus than those with pCAGGS-EGFP (Fig. 3C).

To examine the molecular mechanism by which DCF1 induces apoptosis, we evaluated the changes in the expression of apoptotic proteins, including Bax and BID, both pro-apoptotic proteins and Bcl-2, Mcl-1 and survivin $(27,28)$, anti-apoptotic proteins that become prominently expressed in transformed cell lines and in all of the most common human cancers, such as those of the lung, colon, pancreas, prostate and breast (28). Notably, survivin is a direct inhibitor of 
A
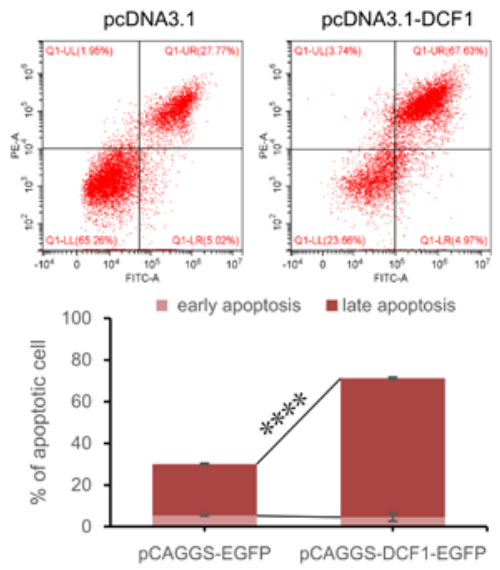

B
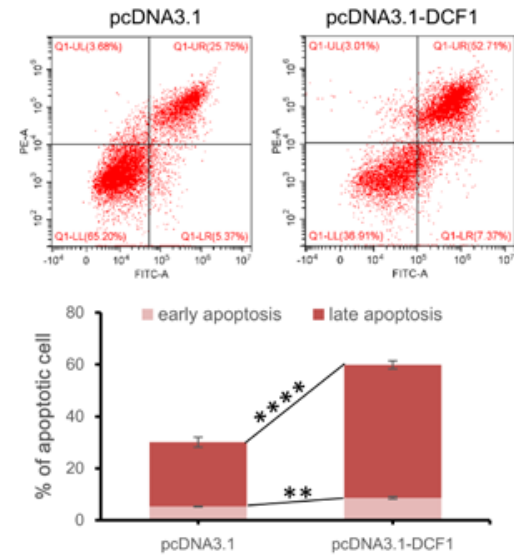

C
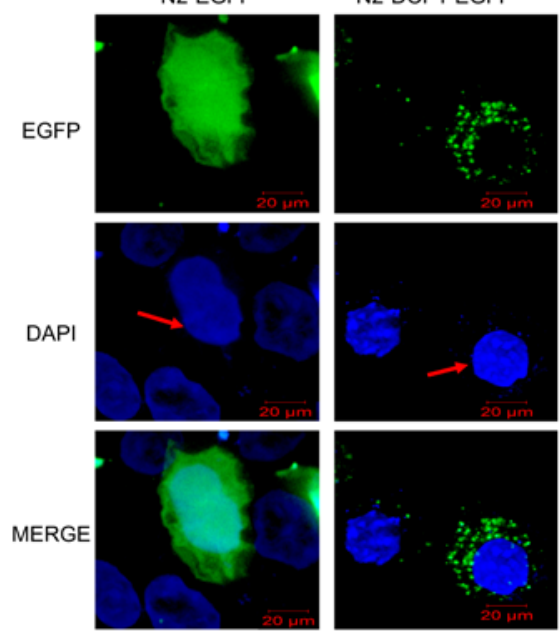

$\mathrm{D}$
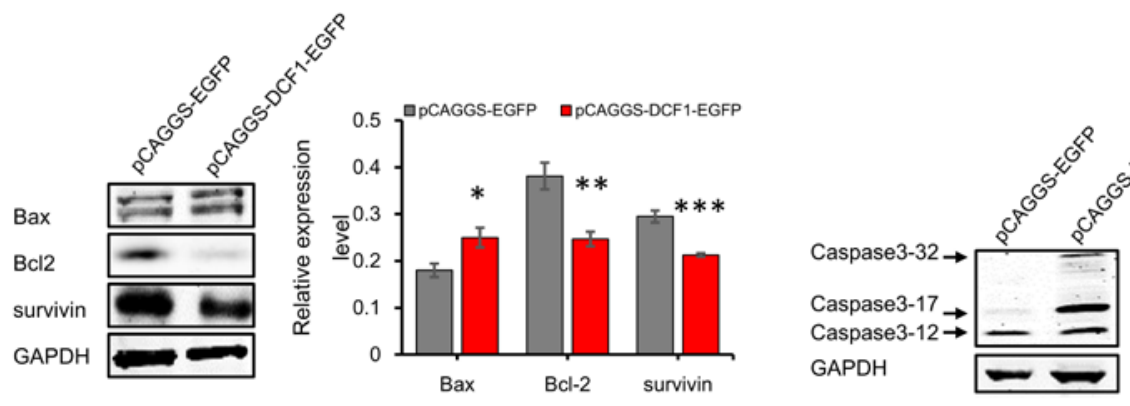

$\mathrm{E}$
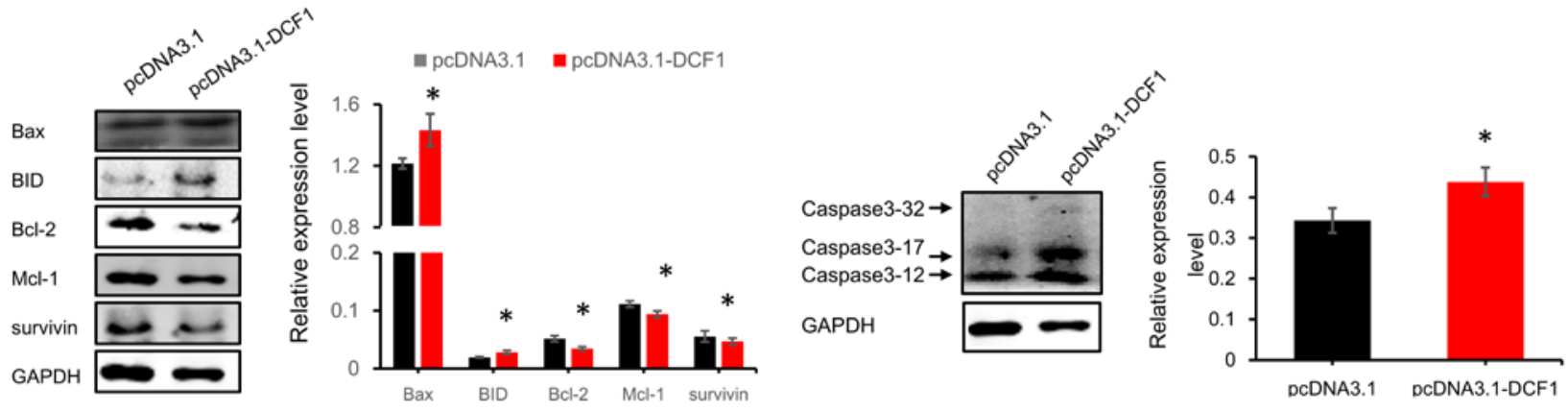

Figure 3. DCF1 promotes NB cells into apoptosis. (A) N2a cells were transfected with pcDNA3.1-myc-his or pcDNA3.1-DCF1-myc-his, respectively, after transfection with dcf1 for $48 \mathrm{~h}$. The effect of DCF1 on apoptosis of N2a was analyzed by Annexin V-APC/7-AAD kit with flow cytometry (upper panel). Quantification confirmed a significant increase in the percentage of late apoptosis $(\mathrm{P}=0.000365)$, while no significant difference in early apoptosis was observed compared with the control group, $\mathrm{n}=6$ (lower panel). (B) SK-N-SH cells were transfected with pcDNA3.1-myc-his or pcDNA3.1-DCF1-myc-his, respectively, after transfection with DCF1 for $48 \mathrm{~h}$. The effect of DCF1 on apoptosis of N2a was analyzed by Annexin V-APC/7-AAD kit with flow cytometry (upper panel). Quantification confirmed a significant increase in the percentage of early $(\mathrm{P}=0.00162)$ and late apoptosis $(\mathrm{P}=0.000352), \mathrm{n}=3$ (lower panel) (C) N2a cells transfected with N2-DCF1-EGFP revealed shrinking morphology and condensed chromosome in nuclei, indicated by white arrows. (D) Western blotting revealed that pro-apoptotic protein (Bax) was upregulated, anti-apoptotic proteins Bcl-2 and survivin were downregulated and apoptotic executor caspase -3 was activated, $n=4$. (E) Western blotting revealed that pro-apoptotic proteins (Bax and BID) were upregulated, anti-apoptotic proteins Bcl-2, Mcl-1 and survivin were downregulated and apoptotic executor caspase-3 was activated, $\mathrm{n}=4 .{ }^{*} \mathrm{P}<0.05,{ }^{* *} \mathrm{P}<0.01,{ }^{* * * *} \mathrm{P}<0.001,{ }^{* * * * *} \mathrm{P}<0.0001$. DCF1, dendritic cell factor 1; NB, neuroblastoma; N2a, Neuro-2a.

caspase-3 and caspase-7 (29) and obstructs the process of apoptosis. Western blot analysis revealed that overexpressed DCF1 led to enhancement of Bax, a decrease of anti-apoptotic proteins Bcl-2 and survivin, and activated apoptotic executor caspase-3 (Fig. 3D). The pro-apoptotic proteins Bax and BID were significantly increased; and the anti-apoptotic proteins Bcl-2, Mcl-1 and survivin were significantly decreased in SK-N-SH cells (Fig. 3E). We also observed that the apoptosis executor caspase-3 was increased and activated with DCF1 in N2A cells (Fig. 3D) and SK-N-SH cells (Fig. 3E). These data revealed that DCF1 induced apoptosis through a caspasedependent pathway in neuroblastoma cells.

DCF 1 inhibits the growth of NB cells in vivo. The in vitro studies indicated that DCF1 may act as a tumor suppressor in NB cells by promoting apoptosis. In the present study, we investigated whether DCF1 could inhibit NB tumorigenesis in vivo. $\mathrm{N} 2 \mathrm{a}$ cells $\left(2 \times 10^{6}\right)$ transfected with either pCAGGS-EGFP or 
A
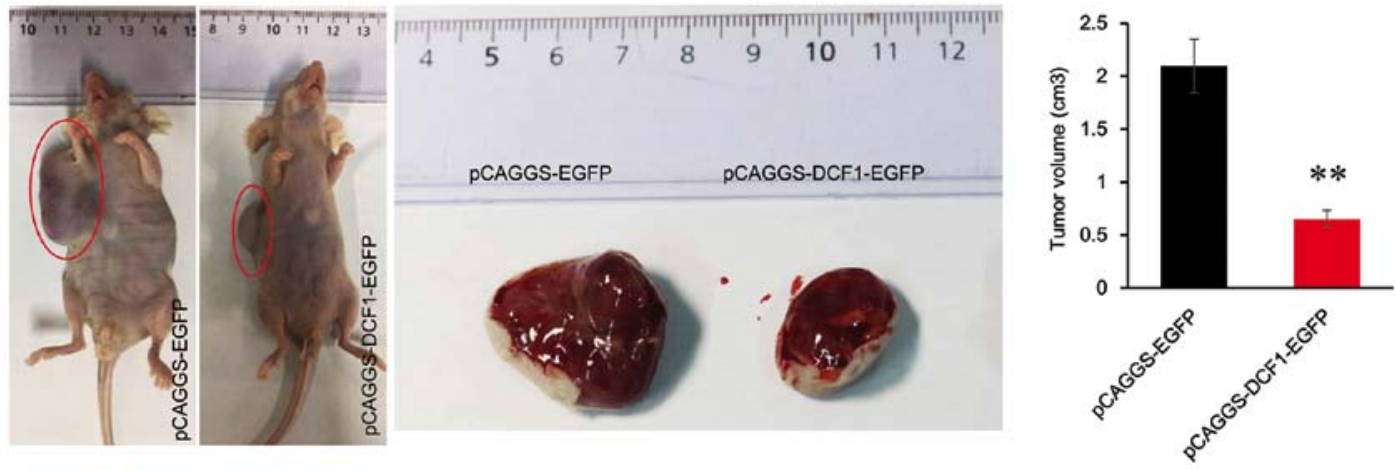

B
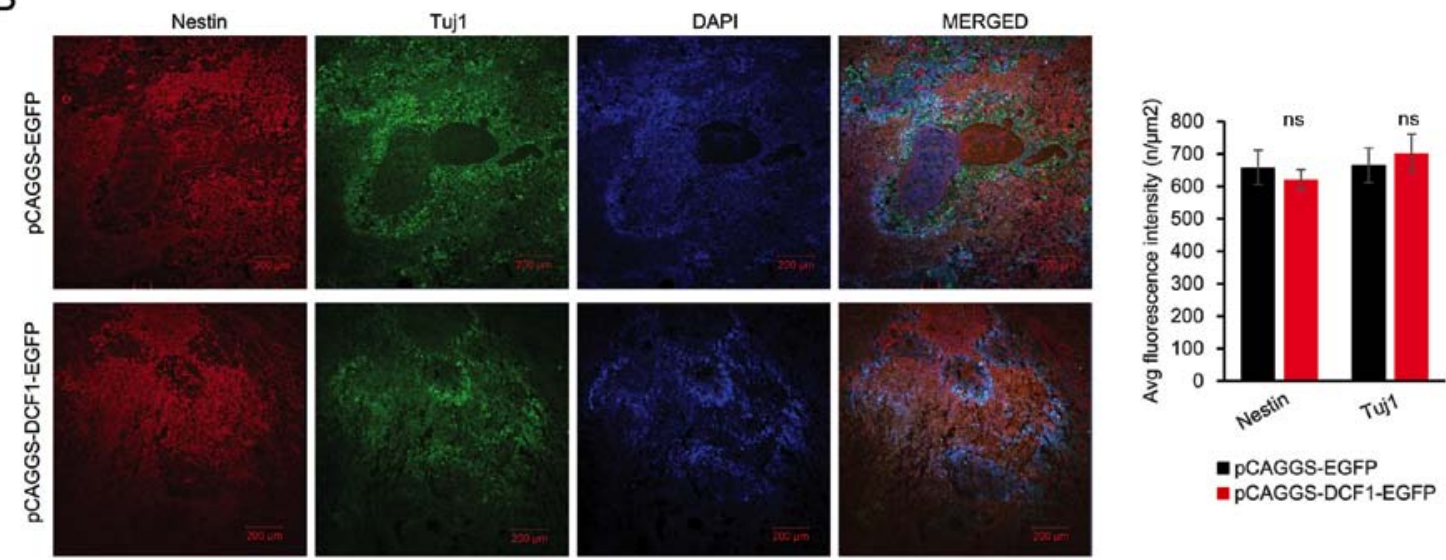

- pCAGGS-DCF1-EGFP

Figure 4. DCF1 reduces tumor growth of neuroblastoma in vivo. (A) Representative images of allograft tumors. Injection of N2a cells expressing DCF1 into nude mice revealed a low tumorigenicity rate and a small tumor volume compared with controls, (4 mice/group). Quantification confirmed a significant decrease in the tumor volume, $\mathrm{n}=4$. (B) Immunofluorescence analysis of allograft tumors revealed that DCF1 had no effect on the differentiation of NB. DCF1, dendritic cell factor 1 ; NB, neuroblastoma; N2a, Neuro-2a. ${ }^{* *} \mathrm{P}<0.01$.

pCAGGS-DCF1-EGFP were subcutaneously injected into the right flank of nude mice ( 8 male 6 -week old mice were used in conducting the cell tumorigenicity experiment; all mice were randomly assigned into two groups, each group consisted of 4 mice) to investigate the relationship between DCF1 and tumorigenesis. As revealed in Fig. 4A, the volume of the tumor with DCF1 was smaller $\left(0.65 \pm 0.08 \mathrm{~cm}^{3}\right)$ than that from the negative control $\left(2.09 \pm 0.25 \mathrm{~cm}^{3}\right)$.

Considering that DCF1 overexpression leads to the maintenance of neural stem cells (NSCs) in an undifferentiated state (18) and the potential for NB cells to terminally differentiate, the expression levels of Nestin and Tuj1 were assessed by immunofluorescence in the tissue slices from the nude mice transplanted with $\mathrm{N} 2$ a cells to examine whether DCF1 induced differentiation in vivo. The results revealed that there was no significant difference between Nestin and Tuj1 in DCF1expressing graft tumors and the control graft tumor (Fig. 4B). Therefore, it was clear that DCF1 inhibited the growth of tumors in vivo but failed to induce differentiation in NB cells.

ERK1/2 signaling pathway is the target of DCF1 in NB cells. To gain deeper insight into the mechanisms by which DCF1 controls cell viability, motility and apoptosis in N2a cells and SK-N-SH cells, we focused on the mitogen-activated protein kinase (MAPK) cascade pathway since previous studies have indicated that the activation or inhibition of the classical
MAPK pathway (or the ERK1/2 signaling pathway) is crucial for controlling tumor cell proliferation, migration, invasion and survival (30-35). We first evaluated the phosphorylation level of ERK1/2 using western blotting. The result revealed that the phosphorylation level of ERK was significantly decreased (Fig. 5A). Notably, downregulating DCF1 by small interfering RNA increased the phosphorylation level of ERK1/2 (Fig. 5B), which suggested that DCF1 inhibited the activation of the ERK1/2 signaling pathway to regulate the apoptosis of NB cells. Subsequently, we detected the upstream regulator of ERK1/2, including Ras, Raf1 and MEK1/2 using immunoblotting. The results revealed that DCF1 significantly decreased the protein expression levels of Ras, Raf1 and MEK1/2 (Fig. 5A), while downregulated expression of DCF1 enhanced the expression of the proteins involved in the ERK1/2 signaling pathway, which indicated the activation of the ERK1/2 pathway (Fig. 5B). These results demonstrated that DCF1 regulated the viability and motility of N2a cells by inhibiting the ERK signaling pathway

\section{Discussion}

NB is a lethal cancer of the brain, and despite great endeavors to improve treatment methods and to understand the molecular mechanisms underlying this disease, the results remain far from our expectations; therefore, it is important and critical 
A
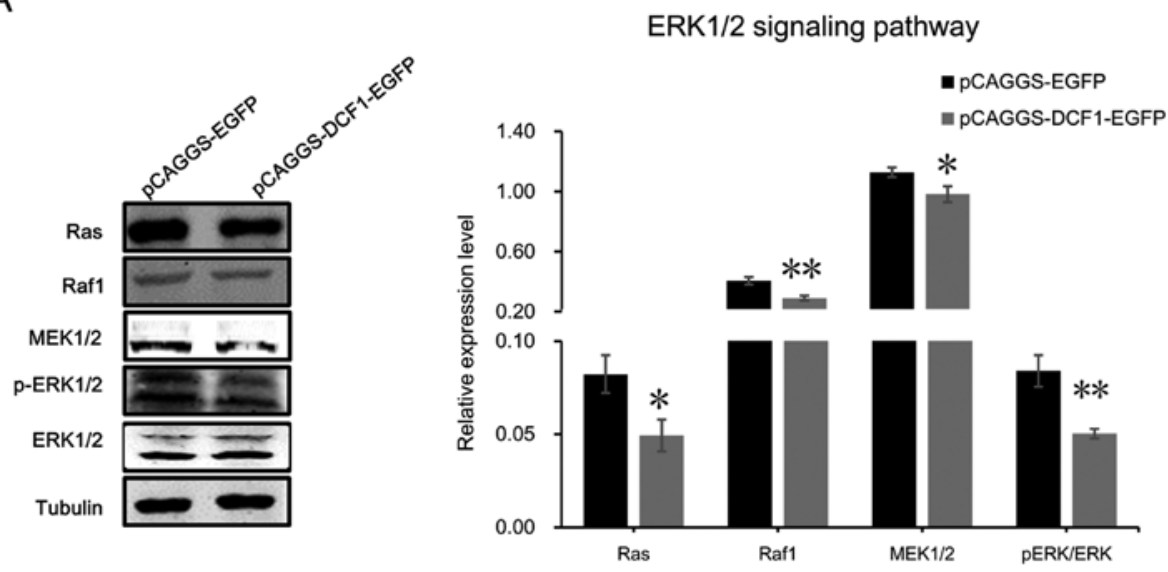

B
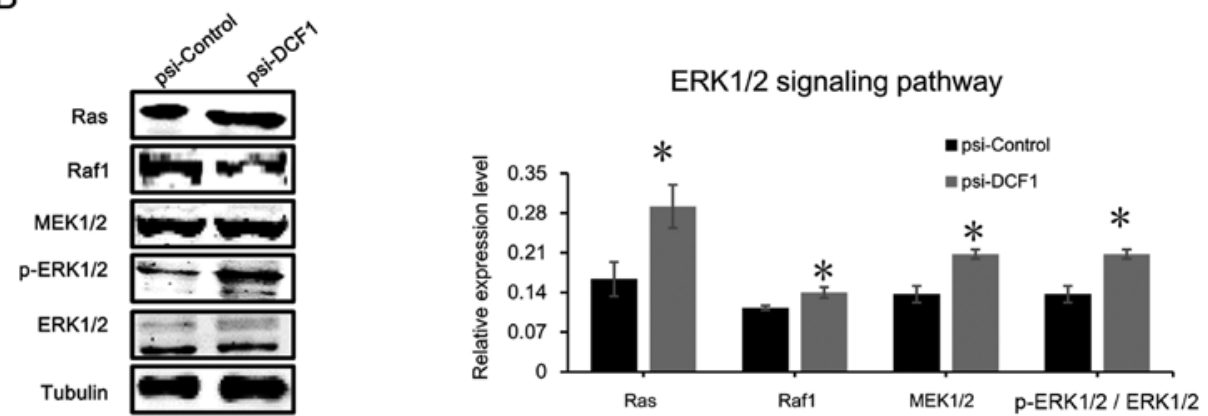

Figure 5. DCF1 targets the ERK1/2 signaling pathway in NB. (A) After transfection of DCF1 for $48 \mathrm{~h}$, N2a cell lysis was detected with ERK1/2 signaling pathway-associated proteins, Ras, Raf1, MEK1/2, p-ERK1/2 and ERK1/2, by western blotting (left panel) and quantification revealed that the expression level of Ras, Raf1, MEK1/2, ERK1/2 and p-ERK1/2 was significantly decreased (right panel), which indicated that overexpressed DCF1 inhibited the ERK1/2 pathway, $n=4$. (B) After transfection of psi-DCF1 to interfere with the expression of DCF1, N2a cell lysis was detected with ERK1/2 signaling pathwayassociated proteins, Ras, Raf1, MEK1/2, p-ERK1/2 and ERK1/2, by western blotting (left panel) and quantification revealed that the expression level of Ras, Raf1, MEK1/2, ERK1/2 and p-ERK1/2 was significantly increased (right panel), which indicated that downregulated DCF1 activated the ERK1/2 pathway, $\mathrm{n}=4 .{ }^{*} \mathrm{P}<0.05,{ }^{* *} \mathrm{P}<0.01$. DCF1, dendritic cell factor $1 ; \mathrm{NB}$, neuroblastoma; N2a, Neuro-2a.

to find a treatment method to eradicate or inhibit NB. In the present study, for the first time, the critical role of DCF1 was demonstrated with regard to the inhibition of proliferation, motility, invasion and apoptosis of $\mathrm{N} 2 \mathrm{a}$ and $\mathrm{SK}-\mathrm{N}-\mathrm{SH}$ cells. We also examined the expression levels of pro-apoptotic proteins Bax and BID, and anti-apoptotic proteins Bcl-2, Mcl-1, survivin and caspase-3. As anticipated, Bcl-2, Mcl-1 and survivin were downregulated, and Bax and BID were upregulated. Finally, DCF1 activated the apoptotic executor caspase-3 and suppressed the growth, but not the differentiation, of tumorigenesis in vivo. Although previous studies have shown that DCF1 can inhibit tumors, the mechanism by which DCF1 functions in cancers has not been fully investigated. In the present, we identified the ERK signaling pathway as a target of DCF1. The Ras/Raf/MEK/ERK cascade played a critical role in the regulation of cell-cycle progression and cell survival, and the aberrant activation of genes within the cascade was involved in the development and progression of nearly one-third of all human cancers (36-39). BRAF or RAS (KRAS, HRAS, or NRAS) mutations could be detected in nearly all tumor cases. $R A S$ mutations are found in $>50 \%$ of all pancreatic cancers, and $B R A F$ mutations, particularly those involved with the Valine 600 codon, are found in very high percentages in several cancers, such as hairy cell leukemia, melanomas and Langerhans cell histiocytosis (40).
Considering that these mutations exhibited wide distribution and played a critical role in several malignancies, great efforts are being made to develop drugs specifically targeting members of this pathway. Unfortunately, although considerable clinical efficacy has emerged by treating cancer patients with Ras, Raf and MEK inhibitors, the acquired resistance from this therapy is one negative side effect. Most cases of resistance were considered ERK dependent, which implies that inhibiting the activity of Ras, Raf, MEK or ERK1/2 could still be an effective method by which to activate the downstream substrate or target genes. Based on this, it has been suggested that ERK1/2 may be an optimal target to conquer the acquired resistance to Ras, Raf and MEK inhibitors (17); therefore, it is now the target in anticancer therapy and it is the subject of more intense studies (41). Our data revealed that DCF1 overexpression reduced the expression or inhibited the activity of Ras, Raf1 and MEK to inhibit activation of the ERK1/2 pathway; therefore, we propose that DCF1 should be regarded as a potential target for treating NB.

DCF1 plays an important role in controlling tumor viability, promoting tumor cell apoptosis, and inhibiting tumorigenesis in vivo, and the data aforementioned demonstrated that DCF1 could be a potential new inhibitor for components of the ERK1/2 signaling pathway and helped our understanding of the molecular mechanism of NB. 


\section{Acknowledgements}

Not applicable.

\section{Funding}

The present study was funded by the National Natural Science Foundation of China (nos. 81271253 and 81471162), the Science and Technology Commission of Shanghai (no. 14JC1402400) and the Key Innovation Project of Shanghai Municipal Education Commission (grant no. 14ZZ090).

\section{Availability of data and materials}

All data used in this study are included in this published article.

\section{Authors' contributions}

TW conceived the study and revised the manuscript. GL designed and conducted the experiments, prepared the figures and wrote the manuscript. RF designed the experiments and analyzed the data. YS cultured the Neuro-2A and SK-N-SH cancer cells and conducted the transfection experiment. LZ fed the BALB/c athymic nude mice and conducted tumor graft in nude mice. YW performed the cellular tests and the plasmid construction; YC participated in the IHC related experiments and in western blotting. All authors read and approved the manuscript and agree to be accountable for all aspects of the research in ensuring that the accuracy or integrity of any part of the work are appropriately investigated and resolved.

\section{Ethics approval and consent to participate}

All animal procedures were carried out in accordance with the guidelines and regulations of the Shanghai University Ethics Committee.

\section{Patient consent for publication}

Not applicable.

\section{Competing interests}

The authors declare that they have no competing interests.

\section{References}

1. Siegel RL, Miller KD and Jemal A: Cancer Statistics, 2017. CA Cancer J Clin 67: 7-30, 2017.

2. Maris JM, Hogarty MD, Bagatell R and Cohn SL: Neuroblastoma Lancet 369: 2106-2120, 2007.

3. DuBois SG, Kalika Y, Lukens JN, Brodeur GM, Seeger RC, Atkinson JB, Haase GM, Black CT, Perez C, Shimada H, et al: Metastatic sites in stage IV and IVS neuroblastoma correlate with age, tumor biology, and survival. J Pediatr Hematol Oncol 21: 181-189, 1999.

4. Cotterill SJ, Parker L, More L and Craft AW: Neuroblastoma: Changing incidence and survival in young people aged $0-24$ years A report from the North of England Young Persons' Malignant Disease Registry. Med Pediatr Oncol 36: 231-234, 2001.

5. Cohn SL, Pearson AD, London WB, Monclair T, Ambros PF, Brodeur GM, Faldum A, Hero B, Iehara T, Machin D, et al; INRG Task Force: The International Neuroblastoma Risk Group (INRG) classification system: An INRG Task Force report. J Clin Oncol 27: 289-297, 2009.
6. Hanahan D and Weinberg RA: Hallmarks of cancer: The next generation. Cell 144: 646-674, 2011.

7. Yang XH, Tang F, Shin J and Cunningham JM: A c-Mycregulated stem cell-like signature in high-risk neuroblastoma: A systematic discovery (Target neuroblastoma ESC-like signature). Sci Rep 7: 41, 2017.

8. DeNardo BD, Holloway MP, Ji Q, Nguyen KT, Cheng Y, Valentine MB, Salomon A and Altura RA: Quantitative phosphoproteomic analysis identifies activation of the RET and IGF-1R/IR signaling pathways in neuroblastoma. PLoS One 8: e82513, 2013.

9. Martinez MA, Ubeda A, Cid MA and Trillo MA: The proliferative response of NB69 human neuroblastoma cells to a $50 \mathrm{~Hz}$ magnetic field is mediated by ERK1/2 signaling. Cell Physiol Biochem 29: 675-686, 2012.

10. Vieira GC, Chockalingam S, Melegh Z, Greenhough A, Malik S, Szemes M, Park JH, Kaidi A, Zhou L, Catchpoole D, et al: LGR5 regulates pro-survival MEK/ERK and proliferative Wnt/ $\beta$ catenin signalling in neuroblastoma. Oncotarget 6: 40053-40067, 2015.

11. Vujic I, Posch C, Sanlorenzo M, Yen AJ, Tsumura A, Kwong A, Feichtenschlager V, Lai K, Arneson DV, Rappersberger K, et al: Mutant NRASQ61 shares signaling similarities across various cancer types--potential implications for future therapies. Oncotarget 5: 7936-7944, 2014.

12. Pugh TJ, Morozova O, Attiyeh EF, Asgharzadeh S, Wei JS, Auclair D, Carter SL, Cibulskis K, Hanna M, Kiezun A, et al: The genetic landscape of high-risk neuroblastoma. Nat Genet 45: 279-284, 2013.

13. Eleveld TF, Oldridge DA, Bernard V, Koster J, Colmet Daage L, Diskin SJ, Schild L, Bentahar NB, Bellini A, Chicard M, et al: Relapsed neuroblastomas show frequent RAS-MAPK pathway mutations. Nat Genet 47: 864-871, 2015.

14. Schramm A, Köster J, Assenov Y, Althoff K, Peifer M, Mahlow E, Odersky A, Beisser D, Ernst C, Henssen AG, et al: Mutational dynamics between primary and relapse neuroblastomas. Nat Genet 47: 872-877, 2015.

15. Roberts PJ and Der CJ: Targeting the Raf-MEK-ERK mitogenactivated protein kinase cascade for the treatment of cancer. Oncogene 26: 3291-3310, 2007.

16. Montagut $\mathrm{C}$ and Settleman J: Targeting the RAF-MEK-ERK pathway in cancer therapy. Cancer Lett 283: 125-134, 2009.

17. Yu Z, Ye S, Hu G, Lv M, Tu Z, Zhou K and Li Q: The RAF-MEK-ERK pathway: Targeting ERK to overcome obstacles to effective cancer therapy. Future Med Chem 7: 269-289, 2015.

18. Wang L, Wang J, Wu Y, Wu J, Pang S, Pan R and Wen T: A novel function of dcf1 during the differentiation of neural stem cells in vitro. Cell Mol Neurobiol 28: 887-894, 2008.

19. Li X, Feng R, Huang C, Wang H, Wang J, Zhang Z, Yan H and Wen T: MicroRNA-351 regulates TMEM 59 (DCF1) expression and mediates neural stem cell morphogenesis. RNA Biol 9: 292-301, 2012.

20. Xie Y, Li Q, Yang Q, Yang M, Zhang Z, Zhu L, Yan H, Feng R, Zhang S, Huang C, et al: Overexpression of DCF1 inhibits glioma through destruction of mitochondria and activation of apoptosis pathway. Sci Rep 4: 3702, 2014.

21. Liu Q, Feng R, Chen Y, Luo G, Yan H, Chen L, Lin R, Ding Y, Wen T: Dcf1 triggers dendritic spine formation and facilitates memory acquisition. Mol Neurobiol 55: 763-775, 2017.

22. Liu Q, Chen Y, Li Q, Wu L and Wen T: Dcf1 regulates neuropeptide expression and maintains energy balance. Neurosci Lett 650: 1-7, 2017.

23. Xie Y, Li Q, Yang Q, Yang M, Zhang Z, Zhu L, Yan H, Feng R, Zhang S, Huang C, et al: Overexpression of DCF1 inhibits glioma through destruction of mitochondria and activation of apoptosis pathway. Sci Rep 4: 3702, 2014. https://doi.org/10.1038/ srep03702.

24. Kim MS, Yu JM, Kim HJ, Kim HB, Kim ST, Jang SK, Choi YW, Lee DI and Joo SS: Ginsenoside Re and Rd enhance the expression of cholinergic markers and neuronal differentiation in Neuro-2a cells. Biol Pharm Bull 37: 826-833, 2014.

25. Weibel S, Hofmann E, Basse-Luesebrink TC, Donat U, Seubert C, Adelfinger M, Gnamlin P, Kober C, Frentzen A, Gentschev I, et al: Treatment of malignant effusion by oncolytic virotherapy in an experimental subcutaneous xenograft model of lung cancer. J Transl Med 11: 106, 2013. https://doi.org/10.1186/1479-587611-106.

26. Pagano M, Pepperkok R, Verde F, Ansorge W and Draetta G: Cyclin $\mathrm{A}$ is required at two points in the human cell cycle. EMBO J 11: 961-971, 1992. 
27. Chipuk JE, Fisher JC, Dillon CP, Kriwacki RW, Kuwana T and Green DR: Mechanism of apoptosis induction by inhibition of the anti-apoptotic BCL-2 proteins. Proc Natl Acad Sci USA 105: 20327-20332, 2008.

28. Ambrosini G, Adida C and Altieri DC: A novel anti-apoptosis gene, survivin, expressed in cancer and lymphoma. Nat Med 3: 917-921, 1997.

29. Shin S, Sung BJ, Cho YS, Kim HJ, Ha NC, Hwang JI, Chung CW, Jung YK and $\mathrm{Oh} \mathrm{BH}$ : An anti-apoptotic protein human survivin is a direct inhibitor of caspase-3 and-7. Biochemistry 40: 1117-1123, 2001.

30. Lavoie JN, L'Allemain G, Brunet A, Müller R and Pouysségur J: Cyclin D1 expression is regulated positively by the $\mathrm{p} 42 /$ p44MAPK and negatively by the p38/HOGMAPK pathway. J Biol Chem 271: 20608-20616, 1996.

31. Caunt CJ, Sale MJ, Smith PD and Cook SJ: MEK1 and MEK2 inhibitors and cancer therapy: The long and winding road. Nat Rev Cancer 15: 577-592, 2015

32. Liu Y, Zhang X, Yang B, Zhuang H, Guo H, Wei W, Li Y, Chen R, Li Y and Zhang N: Demethylation-induced overexpression of Shc3 drives c-Raf-independent activation of MEK/ERK in HCC. Cancer Res 78: 2219-2232, 2018.

33. Yang $\mathrm{C}$, Zheng $\mathrm{W}$ and $\mathrm{Du} \mathrm{W}$ : CXCR3A contributes to the invasion and metastasis of gastric cancer cells. Oncol Rep 36: 1686-1692, 2016.

34. Hou H, Ge C, Sun H, Li H, Li J and Tian H: Tunicamycin inhibits cell proliferation and migration in hepatocellular carcinoma through suppression of CD44s and the ERK1/2 pathway. Cancer Sci 109: 1088-1100, 2018.
35. Zhao H, Liu B and Li J: LIM and SH3 protein 1 knockdown suppresses proliferation and metastasis of colorectal carcinoma cells via inhibition of the mitogen-activated protein kinase signaling pathway. Oncol Lett 15: 6839-6844, 2018.

36. Ferreira-Silva GA, Lages CC, Sartorelli P, Hasegawa FR, Soares MG and Ionta M: Casearin D inhibits ERK phosphorylation and induces downregulation of cyclin D1 in HepG2 cells. Toxicol In Vitro 38: 27-32, 2017.

37. Xu X, Wang Q, He Y, Ding L, Zhong F, Ou Y, Shen Y, Liu H and He S: ADP-ribosylation factor 1 (ARF1) takes part in cell proliferation and cell adhesion-mediated drug resistance (CAM-DR). Ann Hematol 96: 847-858, 2017.

38. Yan Y, Wang L, He J, Liu P, Lv X, Zhang Y, Xu X, Zhang L and Zhang Y: Synergy with interferon-lambda 3 and sorafenib suppresses hepatocellular carcinoma proliferation. Biomed Pharmacother. 88: 395-402, 2017.

39. Uehling DE and Harris PA: Recent progress on MAP kinase pathway inhibitors. Bioorg Med Chem Lett 25: 4047-4056, 2015.

40. Wu PK and Park JI: MEK1/2 Inhibitors: Molecular activity and resistance mechanisms. Semin Oncol 42: 849-862, 2015.

41. Serini S and Calviello G: Modulation of Ras/ERK and phosphoinositide signaling by long-chain n-3 PUFA in breast cancer and their potential complementary role in combination with targeted drugs. Nutrients 9: 185, 2017. doi:10.3390/nu9030185.

(7) $\Theta$ This work is licensed under a Creative Commons International (CC BY-NC-ND 4.0) License. 